\title{
NIVELES DE PROTEÍNA PARA CERDOS EN FASE STARTER: UN META-ANÁLISIS
}

\author{
PROTEIN LEVELS FOR NURSERY PIGS: A META-ANALYSIS \\ González, R.M. ${ }^{1 \text {; }}$ Figueroa, V.J.L. ${ }^{1 *}$; Vaquera, H.H. ${ }^{2}$; Sánchez-Torres, M.T.18; \\ Ortega, C.M.E. ${ }^{1 C}$; Cordero, M.J.L. ${ }^{1 D}$; Copado, B.J.M.F. ${ }^{3}$ y Narciso, G.C. ${ }^{4}$
}

\begin{abstract}
'Colegio de Postgraduados. Montecillo. Texcoco. Estado de México. México. *jlfigueroa@colpos.mx; Agonzalez.monica@colpos.mx; Bteresa@colpos.mx; ${ }^{C}$ meoc@colpos.mx; Dcordero@colpos.mx ${ }^{2}$ Programa de Estadística. Colegio de Postgraduados. Montecillo. Texcoco. Estado de México. México. hvaquera@colpos.mx

${ }^{3}$ Departamento de Zootecnia. Universidad Autónoma Chapingo. México-Texcoco. Chapingo. Texcoco. Estado de México. México. fernandocopado@yahoo.es

${ }^{4}$ Colegio de Postgraduados. Congregación Manuel León. Municipio Amatlán de los Reyes. Veracruz. México. cnarciso@colpos.mx
\end{abstract}

\section{Palabras clave adicionales}

Aminoácidos sintéticos. Estrategias de alimentación.

\section{RESUMEN}

Para determinar el nivel óptimo de proteína bruta (PB) se realizó un meta-análisis con datos de siete experimentos con 240 cerdos híbridos de aproximadamente 37 días de edad y peso vivo inicial promedio de $12,57 \mathrm{~kg}$ en fase starter y alojados individualmente. Se evaluó el comportamiento productivo, características de la canal y concentración de urea en plasma. Se realizó análisis de varianza y de regresión utilizando un modelo de superficie de respuesta con el procedimiento RSREG de SAS tomando en cuenta el nivel de PB y el efecto del experimento, año y época del año; se determinaron los niveles óptimos de PB para cada variable (ganancia diaria de peso $=19,25$; grasa dorsal $=18,1$; conversión alimenticia $=17,3$ y urea en plasma $=16,5 \%$ ), los cuales se corroboraron con un experimento adicional utilizando 40 cerdos híbridos en fase starter de 36 días de edad con $11,5 \mathrm{~kg}$ de peso inicial durante 21 días, en un diseño completamente al azar con diez repeticiones por tratamiento. El meta-análisis indicó que la ganancia diaria de peso, ganancia de carne magra y urea en plasma disminuyen al bajar la PB $(p \leq 0,05)$; y la conversión alimenticia mejora cuando se reduce la PB hasta $16,5 \%(p \leq 0,05)$. En el experimento realizado para

\section{AdDitiONAL KEYWORDS}

Crystalline amino acids. Feeding strategies.

corroborar los niveles óptimos de $\mathrm{PB}$, no hubo diferencias en las variables analizadas, excepto para porcentaje de carne magra, que mejoró $(p \leq 0,05)$ cuando se utilizaron 16,5 y $19,25 \%$ PB. Los resultados indican que el nivel óptimo de PBes de $16,5 \%$ para cerdos en fase starter con dietas a base de sorgo-harina de soja adicionadas con AA sintéticos, ya que en este nivel de PB no se afectan las variables productivas, características de la canal y se disminuye la concentración de urea en plasma.

\section{SUMMARY}

To determine the optimum level of crude protein (CP), a meta-analysis with data of seven trials with 240 hybrid nursery pigs individually penned with average body weight $12.57 \mathrm{~kg}$. Growth performance, carcass characteristics, and plasma urea nitrogen concentration were evaluated. ANOVA and regression analysis were performed using a surface response model with RSREG of SAS procedure considering the crude protein (CP) level as well as the effect of trial, year and season; the optimum CP levels were determined to each variable (average daily body gain $=19.25$, dorsal 


\section{GONZÁLEZ ETAL.}

fat $=18.1$, feed:gain ratio $=17.3$ and plasma urea $\mathrm{N}=16.5 \%$ ), and were corroborated with an additional experiment using 40 hybrid nursery pigs with $11.5 \mathrm{~kg}$ of initial weight during $21 \mathrm{~d}$, in a completely randomized design with ten replicates per treatment. The meta-analysis indicated that average daily gain, lean meat gain and plasma urea nitrogen concentration were reduced when $\mathrm{CP}$ was lowered $(p \leq 0.05)$; feed/ gain ratio was improved when CP was reduced $(p \leq 0.05)$. In the experiment conducted to corroborate the optimum $\mathrm{CP}$ levels, there was no difference in the analyzed variables, except the percentage of lean meat, that improved $(\mathrm{p} \leq 0.05)$ when pigs were fed 16.5 and $19.25 \%$ CP. The results indicate that the optimal level of CP is $16.5 \%$ for nursery pigs diets based on sorghum-soybean meal with synthetic AA added, because of at this level of CP, neither production variables nor carcass characteristics were affected and urea concentration in plasma decreased.

\section{INTRODUCCIÓN}

La reducción del nivel de proteína en la dieta con la adición de aminoácidos (AA) sintéticos, en la actualidad, se centra en la reducción del nitrógeno y del olor fétido del excremento. Por cada unidad porcentual de proteína que se reduce en la dieta, disminuyen aproximadamente en $8 \%$ las pérdidas de nitrógeno en las heces, $19 \%$ el amoniaco en el aire y $17,5 \%$ la fetidez (Lewis, 2001). Sin embargo, dietas con baja concentración de proteína pueden reducir el crecimiento en fase starter (Nyachoti et al., 2006; Wellock et al., 2006, 2008), aunque tal efecto puede ser de corta duración. Una reducción en el contenido de proteína bruta (PB) para lechones al destete disminuye el riesgo de diarrea posdestete ya que se reduce su disponibilidad para los agentes patógenos entéricos, incluyendo Escherichia coli enterotoxigénica (Wellock et al., 2008), y disminuye la producción de aminas biogénicas a partir de la fermentación de las proteínas.

Trujillo et al. (2007) observaron que en lechones en fase starter, variando el porcentaje de PB y la concentración de energía metabolizable (EM; Mcal kg ${ }^{-1}$ ), es posible mantener un comportamiento productivo aceptable si se reduce la PB (de 20,6 a 14,38\%) y alrededor de $100 \mathrm{kcal} \mathrm{kg}^{-1} \mathrm{EM}$ en dietas a base de sorgo-harina de soja suplementadas con lisina, metionina, treonina y triptófano comparadas con dietas con cantidades convencionales de nutrientes. Resultados similares se obtuvieron (Figueroa et al., 2003) con dietas a base de maíz-harina de soja con $12 \%$ de PB más lisina, metionina, treonina, triptófano, isoleucina, histidina y valina; mientras que Reynoso et al. (2004) encontraron que lechones de tres semanas de edad alimentados con dieta trigo-harina de soja con $18,4 \%$ PB presentaron un incremento de $19,9 \%$ en el consumo de alimento (CDA) en comparación con dietas que contenían $20,3 \%$ PB, y tuvieron la mejor conversión alimenticia (CA) al compararlos con los que consumieron la dieta testigo.

El meta-análisis es un procedimiento para combinar datos procedentes de estudios independientes, para el análisis cuantitativo, y es particularmente útil cuando los resultados de varios estudios sobre un tema son inconsistentes o en conflicto y cuando el tamaño de muestra es pequeña, lo que limita el poder estadístico de los estudios individuales para detectar las diferencias (De Coster, 2009).

El presente estudio se llevó a cabo para determinar cuantitativamente, por medio de un meta-análisis, la respuesta productiva, las características de la canal y la concentración de urea en plasma de cerdos en fase starter, alimentados con diferentes niveles de proteína; para obtener una ecuación de regresión para determinar el nivel óptimo de $\mathrm{PB}$ en la dieta para la mejor respuesta de las variables mencionadas; y para corroborar los niveles óptimos de PB obtenidos en el meta-análisis.

\section{MATERIAL Y MÉTODOS}

BASE DE DATOS Y DISEÑO EXPERIMENTAL El conjunto de datos utilizados se basó 


\section{META-ANÁLISIS DIETAS CONBAJA PROTEÍNA}

en una línea de investigación sobre la alimentación de cerdos con diferentes niveles de proteína en fase starter. Los datos se encuentran publicados en revistas científicas, en manuscritos en proceso de arbitraje para su publicación, y en material todavía no publicado (tesis de maestría) pero en proceso de elaboración de manuscritos para publicarse como artículos (tabla I). Se recopilaron de estudios realizados desde julio de 2005 a julio de 2011. Los estudios analizados tenían en común evaluar diferentes niveles de proteína en dietas para cerdos en fase starter alimentados con dietas a base de sorgo-harina de soja. Los criterios que se utilizaron en este meta-análisis para el uso de los datos fueron, en primer lugar, que cada experimento debió tener más de una repetición de cada tratamiento, independientemente del tamaño de la muestra. En segundo lugar tenía que haber una clara comparación de los resultados de los cerdos alimentados con una dieta con nivel de proteína estándar contra el desempeño de los cerdos alimentados con dietas que contenían niveles de proteína diferente al estándar. Y, en tercer lugar, el experimento tuvo que ser concurrente, es decir, que los tratamientos de cada experimento se aplicaran al mismo tiempo.

Fue importante que los experimentos contaran con el conjunto de datos completos (originales), para asegurar que las omisiones no introdujeran sesgo y construir una base de datos lo suficientemente grande que fuese insensible a las variaciones en los valores de los distintos experimentos o de la adición o eliminación de los experimentos individuales. Se contó con datos de siete experimentos con un total de 240 cerdos destetados a los 21 días, con edad aproximada de 37 días de edad y un peso vivo inicial promedio de $12,57 \mathrm{~kg}$, siendo cada cerdo la unidad experimental (tabla I). En cada estudio los cerdos se alojaron en corrales individuales de $1,5 \times 1,2 \mathrm{~m}$, equipado con comedero tipo tolva de una boca y bebedero de chupón. En todos los experimentos el agua y el alimento se ofrecieron a libre acceso. La limpieza de corrales e inspección del estado de salud de los cerdos se realizó diariamente. El peso de los cerdos para calcular su ganancia diaria (GDP), el consumo diario de alimento (CDA), y la conversión alimenticia (CA), se obtuvieron semanalmente. El último día de cada experi-

Tabla I. Estudios utilizados para evaluar el comportamiento productivo, características de la canal y concentración de urea en plasma de cerdos en fase starter. (Trials for evaluation of growth performance, carcass characteristics and plasma urea nitrogen concentration of nursery pigs).

\begin{tabular}{lccccccc}
\hline Estudio & Número & Raza & Sexo & PVI & PVF & Días & Publicación \\
\hline Trujillo et al. (2007) & 50 & $\mathrm{~L} \times \mathrm{Y} \times \mathrm{D}$ & $\mathrm{H}$ & 10,22 & 18,50 & 35 & Artículo \\
Zamora et al. (2011) & 40 & $\mathrm{Y} \times \mathrm{D} \times \mathrm{P}$ & $\mathrm{MC}$ & 12,20 & 24,78 & 21 & Artículo \\
Martínez et al. (2012) & 24 & $\mathrm{Y} \times \mathrm{D} \times \mathrm{P}$ & $\mathrm{MC}$ & 12,35 & 26,84 & 28 & Artículo \\
Reyes et al. (2012) & 36 & $\mathrm{~L} \times \mathrm{Y} \times \mathrm{D}$ & $\mathrm{MC}$ & 11,10 & 25,70 & 21 & Artículo \\
Figueroa et al. (2012) & 36 & $\mathrm{Y} \times \mathrm{D} \times \mathrm{P}$ & $\mathrm{MC}$ & 10,60 & 25,60 & 21 & Artículo \\
Salinas-Sánchez (2011) & 30 & $\mathrm{Y} \times \mathrm{D} \times \mathrm{P}$ & $\mathrm{MC}$ & 18,12 & 32,50 & 21 & Tesis-Maestría \\
Salinas-Sánchez (2011) & 32 & $\mathrm{Y} \times \mathrm{D} \times \mathrm{P}$ & $\mathrm{MC}$ & 13,52 & 26,20 & 21 & Tesis-Maestría \\
\hline
\end{tabular}

$\mathrm{PVI}=$ peso vivo inicial; $\mathrm{PVF}=$ peso vivo final; $\mathrm{H}=$ hembras; $\mathrm{MC}=$ machos castrados; $\mathrm{L} \times \mathrm{Y} \times \mathrm{D}=$ Landrace $\times$ Yorkshire $\times$ Duroc; $Y \times D \times P=$ Yorkshire $\times$ Duroc $\times$ Pietrain.

Las dietas utilizadas en todos los experimentos fueron a base de sorgo-harina de soja.

En todos los experimentos las variables de respuesta fueron: GDP, CDA, CA, peso final, GCM, GD, AML, PCM y urea en plasma. 


\section{GONZÁLEZ ETAL.}

mento se tomaron muestras de sangre mediante punción en la vena cava utilizando un tubo vacutainer con heparina; las muestras se colocaron en hielo hasta centrifugarse durante $20 \mathrm{~min}$ a $2500 \mathrm{rpm}(1,286 \mathrm{~g})$ para separar el plasma, que se colocó en tubos de polipropileno y se congeló a $-20{ }^{\circ} \mathrm{C}$ hasta realizar las determinaciones de urea en plasma por espectrofotometría de absorción atómica (Chaney y Marbach, 1962).

El día primero y último de la etapa de cada experimento se midió grasa dorsal (GD) y el área del músculo Longissimus (AML) con ultrasonidos de tiempo real Sonovet 600 (Medison, Inc., Cypress, California, USA). Con estos datos y los de peso inicial y final (PF), se calculó la ganancia de carne magra (GCM) y el porcentaje de carne magra (PCM) en la canal, con la ecuación del NPPC (1991).

\section{ANÁLISIS ESTADÍ́STICO}

Para investigar el efecto de los factores sobre las variables de respuesta se utilizó el siguiente modelo de efectos:

$$
\mathrm{Y}_{\mathrm{ijk}}=\mu+\mathrm{T}_{\mathrm{i}}+\beta_{\mathrm{j}}+\alpha_{\mathrm{k}}+\varepsilon_{\mathrm{ijk}}
$$

donde:

$\mu=$ media general;

$\mathrm{T}_{\mathrm{i}}=$ efecto del $\mathrm{i}$-ésimo experimento;

$\beta_{\mathrm{i}}=$ efecto del $\mathrm{j}$-ésima época del año;

$\alpha_{k}=$ efecto del k-ésimo aditivo;

$\varepsilon_{\mathrm{ijk}}=$ error experimental asociado con cada medición.

Del análisis de varianza se obtuvieron los promedios del comportamiento productivo dentro de los cuales se consideró: CDA, GDP, CA, GCM y PF y en las características de la canal se contempló: GD, PCM, AML y urea.

Posteriormente, se realizó un análisis de regresión lineal usando un modelo de superficie de respuesta con el comando RSREG de SAS (2009); esta es una técnica de optimización basada en planeamientos factoriales para obtener el nivel óptimo de proteína para las diferentes variables productivas, características de la canal y urea.

\section{EXPERIMENTO PARA CORROBORAR EL META-ANÁLISIS}

Una vez que se analizaron los datos de los experimentos con el meta-análisis, se realizó un experimento para corroborar los resultados de las regresiones de donde se obtuvo el nivel de proteína óptimo para cada variable productiva, características de la canal y concentración de urea en plasma. Los niveles de proteína utilizados en el experimento de corroboración, fueron los de las variables que presentaron mayores valores para $\mathrm{R}^{2}$ en el meta-análisis y correspondieron a los siguientes: $\operatorname{GDP}\left(\mathrm{R}^{2}, 0,593\right.$; nivel óptimo de $\mathrm{PB}, 19,25 \%), \mathrm{CA}\left(\mathrm{R}^{2}, 0,593\right.$; nivel óptimo de $\mathrm{PB}, 17,3 \%)$, y GD ( $\mathrm{R}^{2}, 0,994$; nivel óptimo de PB, 18,1\%). Para urea en plasma, la ecuación de regresión había determinado un contenido óptimo de PB de $12,1 \%$. Sin embargo, este nivel se consideró demasiado bajo para cerdos en fase starter, por lo que se desechó y en su lugar se incluyó el nivel de 16,5 \% PB, con el cual se había observado consistentemente que se mantenía la respuesta productiva de los cerdos en fase starter en la mayoría de los experimentos cuyos datos se utilizaron en el meta-análisis (tabla II).

El experimento se efectuó en la Unidad Porcina de la Granja Experimental del Colegio de Postgraduados, en el Campus Montecillo, Texcoco México. Se utilizaron 20 cerdos machos castrados y 20 hembras (Yorkshire $\times$ Duroc $\times$ Pietrain) de 36 días de edad con un peso promedio inicial de $11,5 \mathrm{~kg}$ durante 21 días en fase starter. El diseño experimental fue completamente al azar, para evaluar cuatro concentraciones de proteína cruda, con diez repeticiones por tratamiento. El modelo estadístico fue el siguiente:

$$
Y_{i j}=\mu+A_{i}+\varepsilon_{i j}
$$

donde:

$Y_{i j}=$ variable de respuesta en la j-ésima repetición 


\section{META-ANÁLISIS DIETAS CONBAJA PROTEÍNA}

Tabla II. Composición de las dietas experimentales para cerdos en fase starter. (Composition of experimental diets for nursery pigs).

\begin{tabular}{|c|c|c|c|c|c|}
\hline Tratamiento & $\mathrm{T} 1$ & $\mathrm{~T} 2$ & T3 & $\mathrm{T} 4$ & NRC \\
\hline \multicolumn{6}{|l|}{ Ingrediente, \% } \\
\hline Sorgo & 63,020 & 66,142 & 68,314 & 70,486 & \\
\hline Harina de soja & 30,628 & 27,269 & 24,933 & 22,597 & \\
\hline Aceite crudo de soja & 2,592 & 2,630 & 2,656 & 2,682 & \\
\hline Ortofosfatos & 1,530 & 1,550 & 1,565 & 1,579 & \\
\hline $\mathrm{CaCO}_{3}$ & 1,071 & 1,075 & 1,078 & 1,081 & \\
\hline Sal común & 0,350 & 0,350 & 0,350 & 0,350 & \\
\hline Vitaminas $^{2}$ & 0,150 & 0,150 & 0,150 & 0,150 & \\
\hline Antibiótico (oxitetraciclina) & 0,100 & 0,100 & 0,100 & 0,100 & \\
\hline Minerales $^{3}$ & 0,100 & 0,100 & 0,100 & 0,100 & \\
\hline Tripto + Plus ${ }^{4}$ & 0,353 & 0,459 & 0,534 & 0,608 & \\
\hline DL-Metionina ${ }^{5}$ & 0,064 & 0,091 & 0,109 & 0,128 & \\
\hline L-Treonina ${ }^{6}$ & 0,038 & 0,080 & 0,109 & 0,138 & \\
\hline Total & 100 & 100 & 100 & 100 & \\
\hline \multicolumn{6}{|l|}{ Análisis calculado } \\
\hline Energía metabolizable, Mcal kg-1 & 3,265 & 3,265 & 3,265 & 3,265 & 3,265 \\
\hline Proteína bruta, \% & 19,25 & 18,1 & 17,3 & 16,5 & 20,9 \\
\hline Calcio, \% & 0,80 & 0,80 & 0,80 & 0,8 & 0,8 \\
\hline Fósforo total, \% & 0,65 & 0,642 & 0,636 & 0,63 & 0,662 \\
\hline Fósforo disponible, \% & 0,40 & 0,40 & 0,40 & 0,40 & 0,40 \\
\hline Lisina, \% & 1,10 & 1,10 & 1,10 & 1,10 & 1,10 \\
\hline Treonina, \% & 0,63 & 0,63 & 0,63 & 0,63 & 0,65 \\
\hline Triptófano, \% & 0,247 & 0,249 & 0,251 & 0,253 & 0,244 \\
\hline Metionina + cistina, $\%$ & 0,58 & 0,58 & 0,58 & 0,58 & 0,58 \\
\hline Arginina, \% & 1,132 & 1,038 & 0,973 & 0,907 & 1,266 \\
\hline Histidina, \% & 0,438 & 0,409 & 0,389 & 0,369 & 0,479 \\
\hline Isoleucina, \% & 0,80 & 0,749 & 0,714 & 0,679 & 0,872 \\
\hline Leucina, \% & 1,65 & 1,589 & 1,546 & 1,503 & 1,738 \\
\hline Valina, \% & 0,847 & 0,799 & 0,766 & 0,733 & 0,915 \\
\hline Fenilalanina + tirosina & 1,549 & 1,461 & 1,400 & 1,340 & 1,673 \\
\hline \multicolumn{6}{|l|}{ Análisis determinado } \\
\hline Proteína bruta, \% & 25,62 & 24,17 & 22,83 & 21,79 & \\
\hline Calcio, \% & 0,95 & 1,23 & 1,06 & 1,37 & \\
\hline Fósforo total, \% & 0,74 & 0,74 & 0,69 & 0,88 & \\
\hline
\end{tabular}

$\mathrm{NRC}=$ necesidades en nutrientes para cerdos en fase starter $\left(10-20 \mathrm{~kg}\right.$ de peso vivo); ${ }^{2} \mathrm{Cada} \mathrm{kg}$ de alimento aportó: Vit., $15000 \mathrm{UI}$; Vit.D3, $2500 \mathrm{UI}$; Vit E, 37,5 UI; Vit. K, 2,5 mg; tiamina, 2,25 mg; riboflavina, $6,25 \mathrm{mg}$; niacina, $50 \mathrm{mg}$; cianocobalamina, $0,0375 \mathrm{mg}$; biotina, $0,13 \mathrm{mg}$; ácido fólico, 1,25 mg; ácido pantoténico, $20 \mathrm{mg}$; colina, $563 \mathrm{mg} .{ }^{3}$ Aportó por kg de alimento: $\mathrm{Fe}, 150 \mathrm{mg} ; \mathrm{Zn}, 150 \mathrm{mg} ; \mathrm{Mn}, 150 \mathrm{mg}$; Cu, $10 \mathrm{mg}$; Se, 0,15 mg; I, 0,9 mg; Cr, 0,2 mg. ${ }^{4}$ Tripto-Plus contiene: lisina, 55,3\%; treonina, 0,15\%; triptófano,

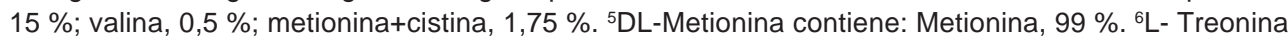
contiene: Treonina, $98 \%$.

Archivos de zootecnia vol. 63, núm. 242, p. 319. 


\section{GONZÁLEZ ETAL.}

del i-ésimo contenido de A;

$\mu=$ media general;

$A_{i}=$ efecto del i-ésimo contenido de proteína; $\varepsilon_{\mathrm{ij}}=$ error experimental.

Las dietas (tabla II) se formularon con base en sorgo-harina de soja adicionadas con L- lisina $\cdot \mathrm{HCl}$, L-treonina, DL-metionina y L-triptófano sintéticos, agregados hasta alcanzar los requerimientos nutricionales para las etapa de iniciación (NRC, 1998). Los cerdos recibieron las mismas condiciones de alojamiento y manejo que en los experimentos utilizados en el meta-análisis, y las variables analizadas (GDP, CDA, CA, PF, GCM, GD, AML, PCM y UREA) se midieron de igual manera.

\section{RESULTADOS}

\section{META-ANÁLISIS}

Los resultados del meta-análisis se presentan en la tabla III. Para las variables productivas GDP y GCM se observó una disminución de la respuesta al bajar el nivel de proteína en la dieta de 20,9 a $14,5 \%$ $(\mathrm{p} \leq 0,05)$, pero utilizando 16 y $20,5 \%$ se presentó el mayor promedio para GDP (613 $\mathrm{g} \mathrm{d}^{-1}$ y $606 \mathrm{~g} \mathrm{~d}^{-1}$, respectivamente); la GCM no fue diferente con 20,9 y $16,5 \%$ de PB, pero al reducir a 14,5\% disminuyó en $28 \mathrm{~g}$ $\mathrm{d}^{-1}$. El CDA y peso final no fueron afectados por el contenido de $\mathrm{PB}$ en la dieta $(\mathrm{p}>0,05)$. La CA se mejoró $(\mathrm{p} \leq 0,05)$ cuando se utilizaron niveles de 20,5 a 14,5\% PB.

Para las características de la canal (tabla IV) GD, AML y PCM, no se observaron diferencias entre los niveles de proteína analizados $(p>0,05)$. Para el contenido de urea en plasma los resultados son variables aunque hay una disminución $(\mathrm{p} \leq 0,05)$ en la concentración de urea cuando se reduce en 6,4 unidades porcentuales la PB en la dieta. El contenido de 20,9 y 20,5\% PB (considerados niveles estándar de proteína) causaron los mayores valores de UREA respecto a las dietas con baja proteína.

\section{ECUACIONES DE PREDICCIÓN}

Los valores de $\mathrm{R}^{2}$ (tabla $\mathbf{V}$ ) de las ecuaciones de predicción obtenidas mediante el modelo de regresión lineal, indican si la ecuación es la idónea para predecir la respuesta productiva, característica de la canal y concentración de urea en plasma. Las variables que presentaron los mayores valores de $\mathrm{R}^{2}$ y el nivel óptimo de $\mathrm{PB}$ para la máxima respuesta fueron GDP, CA, GD y UREA; con 19,$25 ; 17,30 ; 18,10$ y $12,10 \%$ de $\mathrm{PB}$, respectivamente (figuras $\mathbf{1}$ y $\mathbf{2}$ ).

Tabla III. Meta-análisis del efecto de nivel de proteína bruta incluida en la dieta sobre el comportamiento productivo en cerdos en fase starter. (Meta-analysis of dietary crude protein effect on growth performance of nursery pigs).

\begin{tabular}{|c|c|c|c|c|c|}
\hline PB \% & GDP $\mathrm{g} \mathrm{d}^{-1}$ & CDA $\mathrm{g} \mathrm{d}^{-1}$ & $\mathrm{CA}$ & PFkg & $\mathrm{GCM} \mathrm{g} \mathrm{d}^{-1}$ \\
\hline 14,5 & $552^{\mathrm{b}}$ & 1046 & $1,9116^{b}$ & 25,9022 & $214^{\mathrm{b}}$ \\
\hline 16,0 & $613^{a}$ & 1101 & $1,8593^{b}$ & 26,1271 & $235^{\mathrm{ab}}$ \\
\hline 16,5 & $590^{\mathrm{ab}}$ & 1073 & $1,8296^{b}$ & 26,1591 & $243^{a}$ \\
\hline 17,4 & $591^{\mathrm{ab}}$ & 1048 & $1,7983^{b}$ & 25,3193 & $223^{\mathrm{ab}}$ \\
\hline 18,5 & $583^{a b}$ & 1094 & $1,8298^{b}$ & 26,1465 & $226^{\mathrm{ab}}$ \\
\hline 20,5 & $606^{a}$ & 1074 & $1,8415^{b}$ & 26,1230 & $232^{\mathrm{ab}}$ \\
\hline 20,9 & $598^{\mathrm{ab}}$ & 1085 & $2,1269^{a}$ & 25,9947 & $242^{a}$ \\
\hline
\end{tabular}

a,b Medias de tratamiento o efecto principal con distinta literal indica diferencias $(p \leq 0,05)$. PB $=$ proteína bruta; $\mathrm{GDP}=$ ganancia diaria de peso; $\mathrm{CDA}=$ consumo diario de alimento; $\mathrm{CA}=$ conversión alimenticia; $\mathrm{PF}=$ peso final; GCM= ganancia diaria de carne magra. 


\section{META-ANÁLISIS DIETAS CONBAJA PROTEÍNA}

Tabla IV. Meta-análisis del efecto de nivel de proteína bruta incluida en la dieta sobre las características de la canal y concentración de urea en plasma en cerdos en fase starter. (Meta-analysis of dietary crude protein effect on carcass characteristics and plasma urea nitrogen concentration of nursery pigs).

\begin{tabular}{ccccc}
\hline PB & GD & AML & PCM & Urea \\
\hline 14,5 & 0,3221 & 9,4186 & 44,7903 & $5,5133^{\mathrm{d}}$ \\
16,0 & 0,3205 & 10,102 & 44,9320 & $10,5266^{\mathrm{c}}$ \\
16,5 & 0,2838 & 10,456 & 44,5715 & $5,3611^{\mathrm{d}}$ \\
17,4 & 0,3319 & 9,9904 & 46,1071 & $16,6955^{\mathrm{b}}$ \\
18,5 & 0,2726 & 9,7809 & 44,9227 & $10,8731^{\mathrm{c}}$ \\
20,5 & 0,3099 & 9,7876 & 44,7312 & $22,5921^{\mathrm{a}}$ \\
20,9 & 0,2957 & 10,096 & 44,5992 & $23,6867^{\mathrm{a}}$ \\
\hline
\end{tabular}

abcMedias de tratamiento o efecto principal con distinta literal indica diferencias estadísticas $(p \leq 0,05)$.

$\mathrm{PB}=$ proteína bruta (\%); $\mathrm{GD}=$ grasa dorsal final (cm); $A M L=$ área de músculo Longissimus $\left(\mathrm{cm}^{2}\right)$; $\mathrm{PCM}=$ porcentaje de carne magra; Urea= concentración de urea en plasma $\left(100 \mathrm{~mL}^{-1}\right)$.

Respuesta a los niveles ÓPtimos de PB OBTENIDOS DEL META-ANÁLISIS

No se observaron diferencias para las variables productivas, características de la canal y concentración de urea en plasma (tablas VI y VII), por efecto de los niveles óptimos de PB analizados en el experimento de corroboración. Sólo el PCM (tabla VII) fue mejor $(p \leq 0,05)$ con 16,5 y $19,25 \%$ PB con respecto a los otros dos niveles analizados.

\section{DISCUSIÓN}

\section{COMPORTAMIENTO PRODUCTIVO}

Los resultados del meta-análisis mostraron que al reducir la $\mathrm{PB}$ en seis unidades porcentuales (de 20,5 a 14,5\%) en dietas sorgo-harina de soja adicionadas con aminoácidos sintéticos, los cerdos ganaron menos peso al compararlos con aquellos que recibieron el nivel estándar de proteína bruta $(20,5 \%)$. Una respuesta similar ya había sido observada (Reyna-Santamaría, 2006) al disminuir también seis unidades porcentuales la $\mathrm{PB}$ en la dieta para cerdos en fase starter, lo que también provocó una menor GDP. Esto no coincide con los resultados que se obtuvieron con la línea de investigación que generó los datos originales del meta-análisis (Trujillo et al., 2007; Salinas-Sánchez, 2011; Figueroa et al., 2012; Martínez-Aispuro et al., 2012; Reyes et al., 2012), ya que en ellos se había encontrado

Tabla $\boldsymbol{V}$. Nivel de proteína para obtener la mayor respuesta en el comportamiento productivo, características de la canal y concentración de urea en plasma de cerdos en fase starter. (Protein level to obtain the highest response of growth performance, carcass characteristics and plasma urea nitrogen concentration of nursery pigs).

\begin{tabular}{lclc}
\hline Variable & Nivel PB, $\%$ & \multicolumn{2}{c}{ Ecuación } \\
\hline GDP & 19,25 & $-0,016+0,064(\mathrm{~PB})-0,0016\left(\mathrm{~PB}^{2}\right)$ & $\mathrm{R}^{2}=0,593$ \\
CA & 17,30 & $6,73-0,56(\mathrm{~PB})+0,016\left(\mathrm{~PB}^{2}\right)$ & $\mathrm{R}^{2}=0,593$ \\
Urea & 12,10 & $40,69-5,96(\mathrm{~PB})+0,246\left(\mathrm{~PB}^{2}\right)$ & $\mathrm{R}^{2}=0,816$ \\
PCM & 17,63 & $26,62+2,11(\mathrm{~PB})-0,059\left(\mathrm{~PB}^{2}\right)$ & $\mathrm{R}^{2}=0,271$ \\
AML & 18,19 & $-2,18+1,35(\mathrm{~PB})-0,037\left(\mathrm{~PB}^{2}\right)$ & $\mathrm{R}^{2}=0,276$ \\
GCM & 20,41 & $0,571+0,0175(\mathrm{~PB})-0,0004\left(\mathrm{~PB}^{2}\right)$ & $\mathrm{R}^{2}=0,259$ \\
GD & 18,10 & $0,885-0,064(\mathrm{~PB})+0,0017\left(\mathrm{~PB}^{2}\right)$ & $\mathrm{R}^{2}=0,994$ \\
\hline
\end{tabular}

Nivel $\mathrm{PB}=$ nivel de proteína bruta; $\mathrm{GDP}=$ ganancia diaria de peso; $\mathrm{CA}=$ conversión alimenticia; $\mathrm{PCM}=$ porcentaje de carne magra; $\mathrm{AML}=$ área de músculo Longissimus; $\mathrm{GCM}$ = ganancia diaria de carne magra; $\mathrm{GD}=$ grasa dorsal final. 


\section{GONZÁLEZ ETAL.}

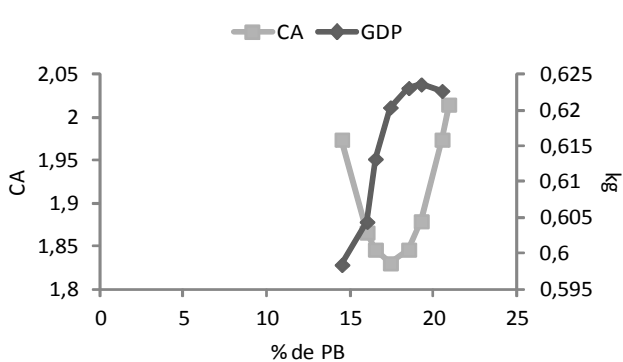

Figura 1. Curva de predicción para la máxima respuesta de GDP $\left(R^{2}=0,593, N P C=\right.$ $19,25 \%)$ y $C A\left(R^{2}=0,593, N P C=17,3 \%\right)$ para cerdos en fase starter con dietas bajas en proteína. (Prediction curve for maximum response of average daily gain $\left(R^{2}=0,593, C P L=\right.$ $19,25 \%)$ and feed conversion $\left(R^{2}=0,593, C P L=\right.$ $17,3 \%)$ for nursery pigs fed low-protein diets).

que la respuesta productiva no se afecta negativamente y que es posible obtener un rendimiento aceptable cuando se reduce la $\mathrm{PB}$ en seis unidades porcentuales en dietas con base en sorgo-harina de soja. Para las variables CDA y $\mathrm{PF}$ no hubo diferencias cuando disminuyó la proteína, lo que coincide con los resultados obtenidos por Trujillo etal.(2007), Salinas-Sánchez(2011),

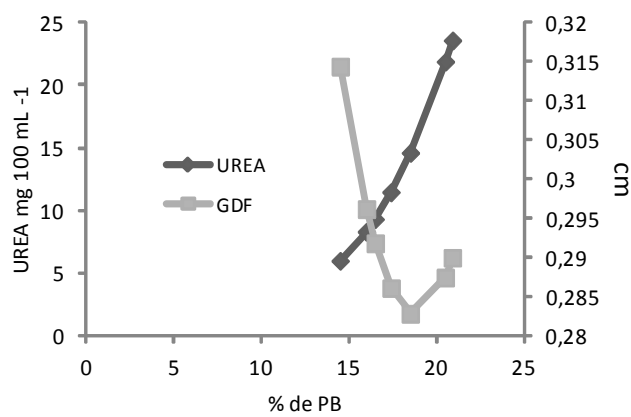

Figura 2. Curva de predicción para la menor respuesta de urea $\left(R^{2}=0,816\right.$, nivel de proteína bruta $=12,10 \%)$ y $G D\left(R^{2}=0,994, N P C=\right.$ $18,10 \%)$ para cerdos en fase starter con dietas bajas en proteína. (Prediction curve for a lower response of plasma urea nitrogen concentration $\left(R^{2}=0.816\right.$, crude protein level $=$ $12.10 \%)$ and backfat thickness $\left(R^{2}=0.994, C P L=\right.$ $18.10 \%$ ) of nursery pigs fed low-protein diets).
Tabla VI. Efecto del nivel óptimo de proteína bruta incluida en la dieta sobre el comportamiento productivo en cerdos en fase starter. (Effect of optimum level of dietary crude protein on growth performance of nursery pigs).

\begin{tabular}{cccccc}
\hline PB & GDP & CDA & CA & PF & GCM \\
\hline 16,50 & 555 & 1087 & 1,965 & 24,00 & 216 \\
17,30 & 592 & 1109 & 1,884 & 24,87 & 214 \\
18,10 & 596 & 1123 & 1,906 & 25,14 & 219 \\
19,25 & 542 & 1056 & 1,956 & 23,09 & 208
\end{tabular}

$\mathrm{PB}=$ proteína bruta $(\%) ; \mathrm{GDP}=$ ganancia diaria de peso $\left(\mathrm{g} \mathrm{d}^{-1}\right) ; \mathrm{CDA}=$ consumo diario de alimento $(\mathrm{g}$ $\left.\mathrm{d}^{-1}\right) ; C A=$ conversión alimenticia; $P F=$ peso final $(\mathrm{kg}) ; \mathrm{GCM}=$ ganancia diaria de carne magra $\left(\mathrm{g} \mathrm{d}^{-1}\right)$.

Figueroa et al. (2012), Martínez-Aispuro et al. (2012) y Reyes et al. (2012); mientras que la CA mejoró y la GCM fue menor cuando se disminuyó la PB en seis unidades porcentuales, lo que coincide con lo encontrado por Reynoso et al. (2004), quienes observaron que la disminución de $\mathrm{PB}$ en la dieta mejora la conversión alimenticia, sin embargo, se debe tener en cuenta que estos inves-

Tabla VII. Efecto del nivel óptimo de proteína bruta incluida en la dieta sobre las características de la canal y concentración de urea en plasma de cerdos en fase starter. (Effect of optimum dietary crude protein level on carcass characteristics and plasma urea nitrogen concentration of nursery pigs).

\begin{tabular}{ccccc}
\hline PB & GD & AML & PCM & Urea \\
\hline 16,50 & 2,900 & 11,214 & $48,165^{\mathrm{a}}$ & 20,82 \\
17,30 & 2,800 & 10,057 & $46,038^{\mathrm{b}}$ & 24,74 \\
18,10 & 2,900 & 10,808 & $46,94^{\mathrm{b}}$ & 21,06 \\
19,25 & 2,727 & 10,923 & $48,620^{\mathrm{a}}$ & 26,13 \\
\hline
\end{tabular}

abMedias de tratamiento o efecto principal con distinta literal indican diferencias $(p \leq 0,05)$.

$\mathrm{GD}=$ grasa dorsal; $\mathrm{AML}=$ área de músculo Longissimus; $\mathrm{PCM}=$ porcentaje de carne magra; Urea $=$ concentración de urea en plasma 


\section{META-ANÁLISIS DIETAS CONBAJA PROTEÍNA}

tigadores utilizaron diferentes ingredientes como fuente de energía y proteína (trigoharina de soja, Reynoso et al., 2004).

\section{Características de LA CANAL}

La disminución de la PB en la dieta no afectó negativamente las características de la canal, lo que coincide con los resultados obtenidos por otros investigadores (Figueroa et al., 2002, 2003, 2004, 2012; Gómez et al., 2002b; Trujillo et al., 2007; SalinasSánchez, 2011; Martínez-Aispuro et al., 2012; Reyes et al., 2012). Sin embargo, hubo una tendencia a aumentar el espesor de GD que podría deberse a un desequilibrio entre AA al agregar lisina a dietas bajas en proteína (Gómez et al., 2002b; Kerr et al., 2003) a la capacidad genética para la retención de tejido magro y al sexo de los cerdos.

\section{CONCENTRACIÓN DE UREA EN PLASMA}

La concentración de urea en plasma disminuyó cuando se redujo el nivel de PB en la dieta, observándose una reducción considerable cuando bajó de 20,9 a 18,5 \% PB $\left(23,686\right.$ a $\left.10,873 \mathrm{mg} \mathrm{dL}^{-1}\right)$, similar a la observada con el cambio de 17,4 a $14,5 \% \mathrm{~PB}$ $\left(16,695\right.$ a $\left.5,513 \mathrm{mg} \mathrm{dL}^{-1}\right)$; esto coincide con los datos obtenidos por otros investigadores (Figueroa et al., 2002, 2003; Gómez et al., 2002a; Kerr et al., 2003; Nyachoti et al., 2006; Trujillo et al., 2007; Zamora et al., 2010; Salinas-Sánchez, 2011; MartínezAispuro et al., 2012; Reyes et al., 2012). Una menor concentración de urea se relaciona con la disminución en la producción de calor metabólico asociado con la síntesis y excreción de urea proveniente del exceso de aminoácidos (Kerr et al., 2003), lo que indica una mejor utilización del nitrógeno por los cerdos alimentados con dietas bajas en proteína suplementadas con AA, por lo que hay una reducción en la excreción de nitrógeno y olor; esta disminución puede ir de 8,5 a $12,5 \%$ por cada unidad porcentual que se reduzca la proteína de la dieta (Ferket et al., 2002; Zervas y Zijlstra, 2002).

\section{NivelEs ÓPTIMOS UTILIZADOS}

Los niveles óptimos de PB, obtenidos mediante las ecuaciones de predicción, indican que para cerdos en iniciación se puede reducir la proteína en la dieta desde 19,25 hasta $16,5 \%$ utilizando sorgo-harina de soja y adicionando AA sintéticos, sin afectar negativamente las variables productivas, las características de la canal y la concentración de urea en plasma; excepto para PCM, que fue mayor cuando se incluyeron 19,25 y $16,5 \%$ PB, en comparación con 17,3 y $18,1 \%$ PB. Debido a que la disminución de la PB desde 20,5 (estándar) hasta 16,5\% (nivel más bajo utilizado) mantiene las variables productivas, este último valor parece ser el adecuado para cerdos en iniciación, el cual es consistente con lo observado en otros trabajos realizados y publicados en esta línea de investigación y utilizados en el meta-análisis.

\section{CONCLUSIONES}

Reducir el contenido de PB en la dieta de cerdos de iniciación hasta $14,5 \%$ afecta adversamente algunas variables productivas como GDP, CA y GCM; sin embargo, no se ven afectadas las características de la canal y hay una disminución de la concentración de urea en plasma produciendo una menor excreción de nitrógeno. El nivel óptimo de PB (16,5 a 19,25\%), sugiere que los cerdos en iniciación se pueden alimentar con dietas bajas en proteína bruta $(16,5 \%)$ con base en sorgo-harina de soja adicionadas con AA sintéticos sin afectar las variables productivas, ni características de la canal y disminuyendo la concentración de urea en plasma.

\section{AGRADECIMIENTOS}

Financiación parcial por el Fideicomiso Revocable de Administración e Inversión $\mathrm{n}^{\circ}$ 167304-2008 del Colegio de Postgraduados. 


\section{GONZÁLEZ ETAL.}

\section{BIBLIOGRAFIA}

Chaney, A.L. and Marbach, E.P. 1962. Modified reagents for determination of urea and ammonia. Clin Chem, 8: 130-132.

De Coster, J. 2009. Meta-analysis notes. http:// www.stat-help.com/notes.html (01/06/2012).

Ferket, P.R.; Van Heugten, E.; Van Kempen, T.A.T.G. and Angel, R. 2002. Nutritional strategies to reduce environmental emissions from nonruminants. J Anim Sci, 80: E168-E182.

Figueroa, J.L.; Lewis, A.J.; Miller, P.S.; Fischer, R.L.; Gómez, S.R. and Diedrichsen, R.M. 2002. Nitrogen metabolism and growth performance of gilts fed standard corn-soybean meal diets or low-crude protein, amino acid-supplemented diets. J Anim Sci, 80: 2911-2919.

Figueroa, J.L.; Lewis, A.J.; Miller, P.S.; Fischer, R.L. and Diedrichsen, R.M. 2003. Growth, carcass traits, and plasma amino acid concentrations of gilts fed low-protein diets supplemented with amino acids including histidine, isoleucine, and valine. J Anim Sci, 81: 1529-1537.

Figueroa, J.L.; Cervantes, M.; Cuca, M. y Méndez, M. 2004. Respuesta de cerdos en crecimiento y finalización a dietas con baja proteína y energía. Agrociencia, 38: 383-394.

Figueroa, J.L.; Estrada, J.; Zamora, V.; Cordero, J.L.; Sánchez-Torres, M.T.; Nieto, R. and Copado, J.M.F. 2012. Digestible lysine levels in lowprotein diets supplemented with synthetic amino acids for nursery, growing, and finishing barrows. Irish J Agr Food Res, 51: 33-44.

Gómez, R.S.; Lewis, A.J.; Miller, P.S. and Chen, H.Y. 2002a. Growth performance, diet apparent digestibility, and plasma metabolite of barrows fed corn-soybean meal diets or low-protein, amino acid supplemented diets at different feeding levels. J Anim Sci, 80: 644-653.

Gómez, R.S.; Lewis, A.J.; Miller, P.S.; Chen, H.Y. and Diedrichsen, R.M. 2002b. Body composition and tissue accretion rates of barrows fed cornsoybean meal diets or low-protein, amino acidsupplemented diets at different feeding levels. J Anim Sci, 80: 654-662.

Kerr, B.J.; Southern, L.L.; Bidner, T.D.; Friesen, K.G. and Easter, R.A. 2003. Influence of dietary protein level, amino acid supplementation, and dietary energy levels on growing-finishing pig performance and carcass composition. J Anim Sci, 81: 3075-3087.

Lewis, A.J. 2001. Amino acids in swine nutrition. In: Lewis, A.J. and Southern, L.L. (Ed.). Swine Nutrition. $2^{\text {nd }}$ ed. CRC Press. New York. pp. 131143.

Martínez-Aispuro, M.; Figueroa-Velasco, J.L.; Zamora-Zamora, V.; Sánchez-Torres, M.T.; Ortega-Cerrilla, M.E.; Cordero-Mora, J.L.; RuizFlores, A. and González-Muñoz, S.S. 2012. Effect of fatty acids source on growth performance, carcass characteristics, plasma urea nitrogen concentration, and fatty acid profile in meat of pigs fed standard- or low-protein diets. Spanish J Agric Res, 10: 993-1004.

NPPC. 1991. National Pork Producers Council. Procedures to evaluate market hogs. $3^{\text {rd }}$ ed. National Pork Producers Council. Des Moines, IA. 17 pp.

NRC. 1998. National Research Council. Nutrient requirements of swine. $10^{\text {th }}$ ed. National Academy Press. Washington DC. 189 pp.

Nyachoti, C.M.; Omogbenigun, F.O.; Rademacher, M. and Blank, G. 2006. Performance responses and indicators of gastrointestinal health in earlyweaned pigs fed low-protein amino acidsupplemented diets. J Anim Sci, 84: 125-134.

Reyes, I.; Figueroa, J.L.; Cobos, M.A.; SánchezTorres, M.T.; Zamora, V. y Cordero, J.L. 2012. Probiótico (Enterococcus faecium) adicionado a dietas estándar y con baja proteína para cerdos. Arch Zootec, 61: 589-598.

Reyna-Santamaría, L. 2006. Balance de nitrógeno, digestibilidad y comportamiento productivo de cerdos alimentados con dietas formuladas con nuevos híbridos de maíz, y con baja proteína adicionada con proteasas. Tesis (D.C.). Programa de Ganadería. Colegio de Postgraduados. México. 161 pp.

Reynoso, E.; Cervantes, M.; Figueroa, J.L. and Cuca, J.M. 2004. Productive response of pigs to low-protein diets added synthetic amino acids and yeast culture. Cuban J Agr Sci, 38: 269275.

Salinas-Sánchez, E. 2011. Respuesta de cerdos en iniciación alimentados con dietas estándar o con baja proteína adicionadas con mananooligosacáridos o nucleótidos. Tesis (M.C.). Pro- 


\section{META-ANÁLISIS DIETAS CONBAJA PROTEÍNA}

grama de Ganadería. Colegio de Postgraduados. México. $67 \mathrm{pp}$.

SAS. 2009. SAS/STAT User's Guide, $2^{\text {nd }}$ ed. SAS Institute. Cary, NC.

Trujillo, J.E.; Figueroa, J.L.; Martínez, M.; Zamora, V.; Cordero, J.L.; Sánchez-Torres, M.T.; Cuca, M. and Cervantes, M. 2007. Plasma urea concentration and growth performance of nursery pigs fed sorghum-soybean meal, lowprotein diets. Agrociencia, 41: 597-607.

Wellock, I.J.; Fortomaris, P.D.; Houdijk, J.G.M. and Kyriazakis, I. 2006. The effect of dietary protein supply on the performance and risk of postweaning enteric disorders in newly weaned pigs. J Anim Sci, 82: 327-335.

Wellock, I.J.; Fortomaris, P.D.; Houdijk, J.G.M. and Kyriazakis, I. 2008. Effects of dietary protein supply, weaning age and experimental enterotoxigenic, Escherichia coli infection in newly weaned pigs: health. Animal, 2: 834842.

Zamora, V.; Figueroa, J.L.; Cordero, J.L.; Rugerio, M.; Reyna, L. and Sánchez-Torres, M.T. 2010. Addition of glucomannans to low-protein diets based on sorghum-soybean meal for growing and finishing pigs. Revista Científica FCV-LUZ, 20: 274-283.

Zamora, V.; Figueroa, J.L.; Reyna, L.; Cordero, J.L.; Sánchez-Torres, M.T. and Martínez, M. 2011. Growth performance, carcass characteristics, and plasma urea nitrogen concentration of nursery pigs fed low-protein diets supplemented with glucomannans or protease. J Appl Anim Res, 39: 1-4.

Zervas, S. and Zijlstra, R.T. 2002. Effects of dietary protein and fermentable fiber on nitrogen excretion patterns and plasma urea in grower pigs. J Anim Sci, 80: 3247-3256. 